\title{
Software Tutorial de apoyo al aprendizaje de los métodos básicos de Análisis Numérico para la solución de problemas de ingeniería
}

\author{
Jesús Alfonso Torres Ortega \\ Escuela de Postgrados, Universidad Nacional Abierta y a Distancia, Bogotá, \\ Colombia.Correo electrónico: jatorresor@unal.edu.co
}

\begin{abstract}
Resumen
Numericals es un software tutorial de apoyo al aprendizaje de las técnicas utilizadas para el Análisis Numérico mediante la programación de los principales métodos numéricos, para la solución de problemas de ingeniería. Los métodos numéricos son técnicas mediante las cuales es posible formular problemas matemáticos de tal forma que puedan resolverse usando operaciones aritméticas. Aunque hay muchos tipos de métodos numéricos, comparten una característica común: invariantemente se debe realizar un buen número de tediosos cálculos aritméticos. No es raro que con el desarrollo de computadoras digitales eficientes y rápidas, el papel de los métodos numéricos en la solución de problemas de ingeniería haya aumentado en forma considerable en los últimos años. Además de proporcionar un aumento en la potencia de cálculo, la disponibilidad general de las computadoras y su asociación con los métodos numéricos ha influido de manera muy significativa en el proceso de la solución de problemas de ingeniería.
\end{abstract}

Palabras claves: Análisis, Numérico, Software, Cálculo, Tutorial.

\begin{abstract}
The software Numericals is a tutorial for support to the learning of the techniques used for the Numerical Analysis mediates the programming of the main numerical methods, for the solution of engineering problems. The numerical methods are technical by means of which it is possible to formulate mathematical problems of such form which they can be solved using arithmetical operations. Although there are many types of numerical methods, share a common characteristic: invariantly a great number of tedious arithmetic calculations are due to make. With the development of efficient and fast digital computers, the paper of the numerical calculates in the solution of engineering problems has increased in considerable form in the last years. Besides to provide an increase in the power of calculation, the general availability of the computers and its association with the numerical methods it has influenced of very significant way in the process of the solution of engineering problems.
\end{abstract}

Key words: Analysis, Numerical, Software, Calculation, Tutorial.

\section{Introducción}

Aunque las soluciones analíticas aún son muy valiosas, tanto para resolver problemas como para proporcionar una mayor comprensión, los métodos numéricos representan alternativas que aumentan en forma considerable la capacidad de confrontar y resolver los problemas; como resultado se dispone de más tiempo para aprovechar las capacidades creativas personales. Por consiguiente, es posible dar más importancia a la formulación de un problema, a la interpretación de la solución y a su incorporación al sistema total, o conciencia "holística". 
Los métodos numéricos reúne dos de los instrumentos más trascendentales en el repertorio de la ingeniería: matemáticas y computadoras. Podemos definir a los métodos numéricos (sin ser muy estrictos) como las matemáticas por computadora. Las buenas técnicas de programación acentúan la destreza para adaptar los conocimientos de los métodos numéricos. En esencia, las potencialidades y restricciones de las técnicas numéricas se advierten mejor cuando se usan estos métodos para resolver los problemas de ingeniería empleando como herramienta una computadora. Los métodos numéricos son un medio para reforzar su comprensión de las matemáticas, ya que una de sus funciones es convertir las matemáticas superiores a operaciones aritméticas básicas, porque profundizan en los temas que de otro modo resultarían oscuros. Adicionalmente, esta alternativa aumenta su capacidad de comprensión y entendimiento en el curso de análisis numérico.

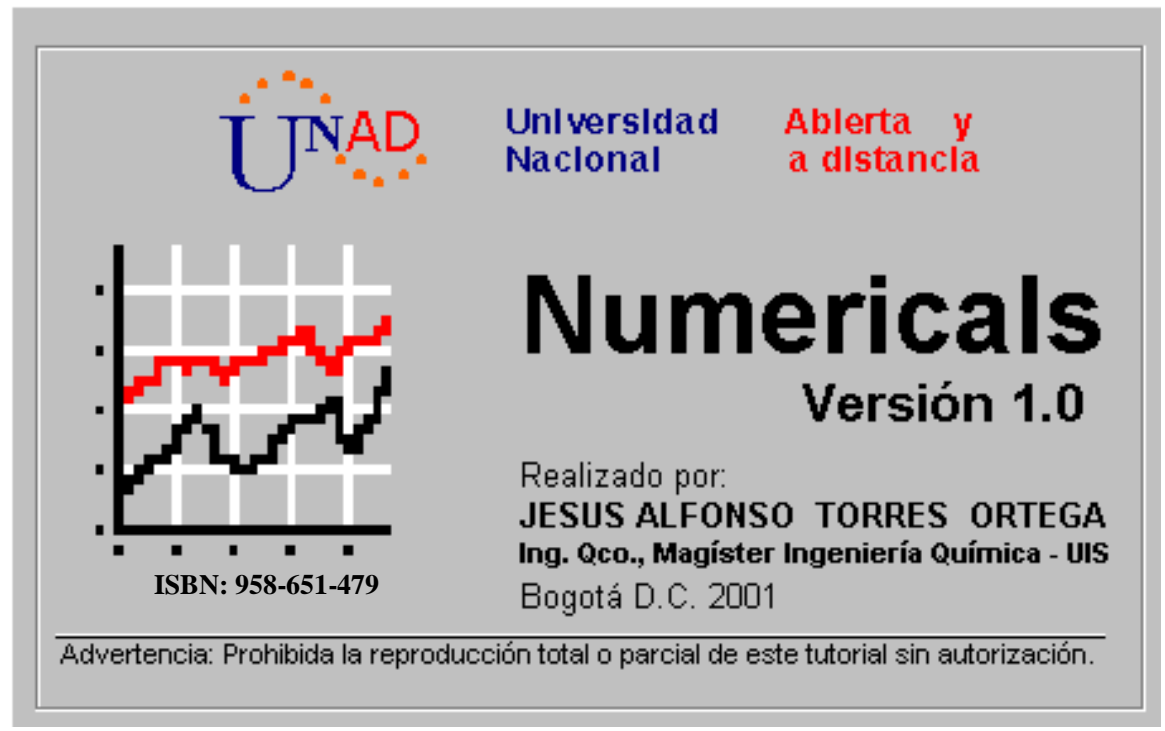

Figura 1. Presentación del software tutorial: Numericals

\section{La interfaz de usuario}

El conjunto completo de pantallas e imágenes utilizadas por el Tutorial se denomina interfaz de usuario del programa. La interfaz incluye todos los menús, cuadros de diálogo, botones, objetos y dibujos que los usuarios verán mientras trabajan con el programa. Si ya está acostumbrado a trabajar con aplicaciones basadas en Windows, reconocerá la mayoría de los elementos de la interfaz de usuario. Los menús contienen muchas de las ordenes disponibles en una aplicación basada en Windows; los cuadro de diálogo, los botones y el puntero del ratón ayudarán al usuario a introducir información en el programa; y las ventanas o formularios y barras de desplazamiento permitirán al usuario ver y recorrer la información que aparece en la pantalla. 


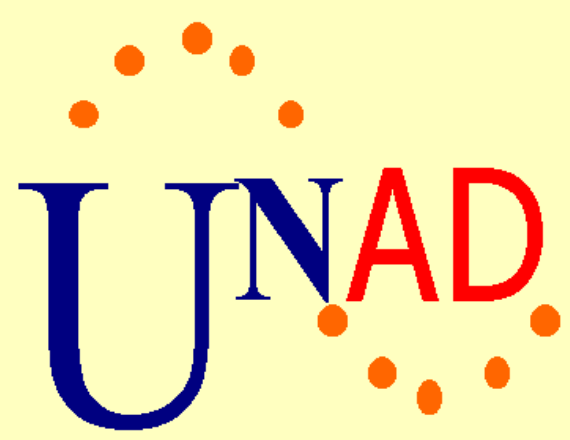

\section{Universidad Abierta y Nacional a distancia}

\section{Salir}

Fecha: 16/12/2001 Numericals v1.0 Bienvenido a su software tutorial de la asignatura Análisis Numérico. Hora: 00:42:40

Figura 2. Interfaz de usuario de ingreso a los métodos numéricos: Raíces de ecuaciones, ajuste de curvas, integración, ecuaciones simultáneas y ecuaciones diferenciales.

Las teclas de acceso de una orden es la tecla del teclado que el usuario puede pulsar para ejecutar la orden. Cuando el usuario abre un menú en tiempo de ejecución, la tecla de acceso de una orden está indicada por una letra subrayada en el nombre de la orden.

\section{Funciones operacionales}

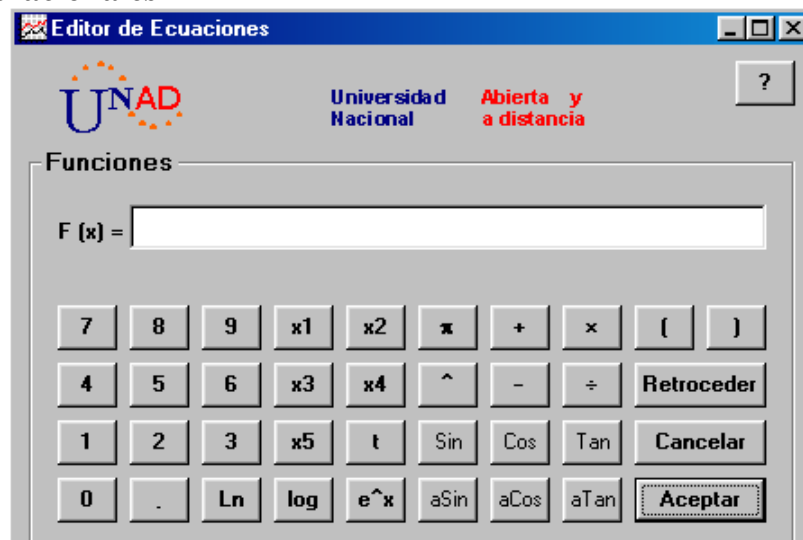

Figura 3. Editor de ecuaciones para ingreso de las funciones $\mathrm{F}(\mathrm{x})$ para los cálculos numéricos. 


\section{Sistema de ayuda interactiva}

Numericals incluye una referencia interactiva que podrá utilizar para aprender más sobre el entorno del software tutorial, sobre los métodos numéricos y sobre el procedimiento adecuado para encontrar la solución en cada caso. El acceso a la información de ayuda se puede realizar de varias formas: El sistema de ayuda empieza con las indicaciones básicas para guiarlo al método en cuestión.

El sistema de ayuda interactiva está diseñado para resolver las posibles inquietudes acerca del manejo correcto del tutorial de Análisis Numérico: Numericals. Una mirada al teclado del «Editor de Ecuaciones» revela que los caracteres y símbolos están localizados sobre las teclas. Estos sé accesan haciendo Click sobre la tecla requerida. Haga Click en el botón comando «Editar», para llamar al formulario compuesto por un conjunto de botones comandos, utilizados para insertar datos o funciones en una caja de texto (pantalla), para los métodos numéricos que lo requieran y cuya configuración se muestra en la figura 4.

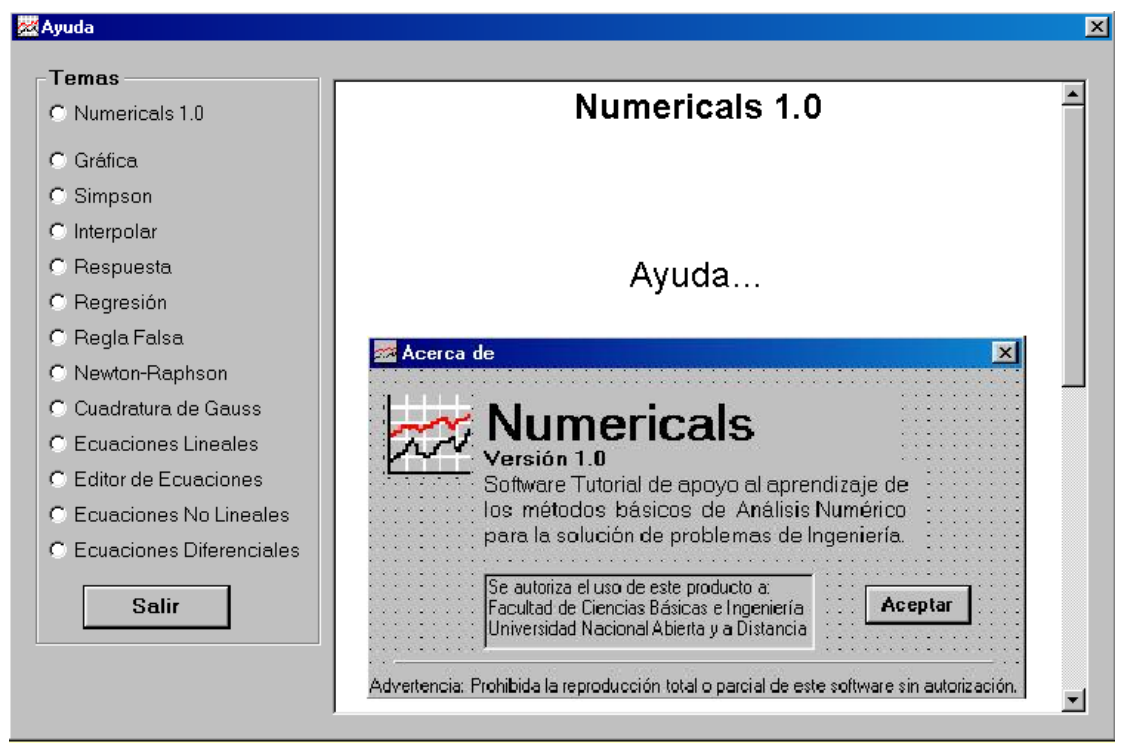

Figura 4. Ventana de presentación para el ingreso al manual de usuario.

\section{Descripción de los formularios presentados para la solución de los principales métodos numéricos}

\section{Raíces de funciones}

Uno de los problemas más básicos del análisis numérico: el problema de la búsqueda de raíces, consiste en encontrar los valores de las variable x que satisfacen la ecuación $\mathrm{F}(\mathrm{x})$ $=0$, para una función $\mathrm{f}$ dada. A una solución de este problema se le llama un cero de $\mathrm{F} o$ una raíz de $\mathrm{F}(\mathrm{x})=0$. Los métodos numéricos presentados se usan para encontrar aproximaciones a las soluciones de ecuaciones cuya solución exacta no se puede encontrar por métodos algebraicos. 


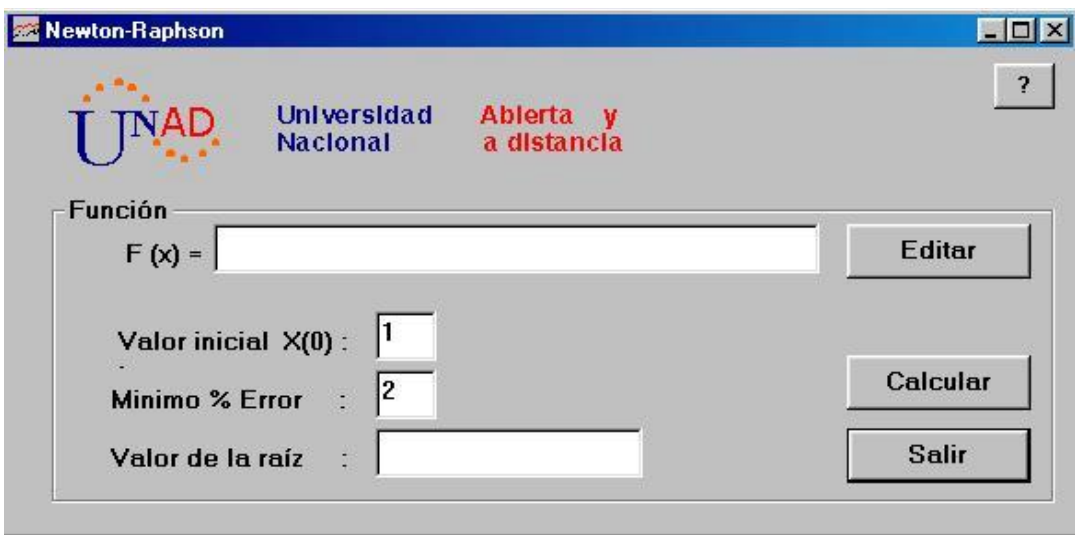

(a)

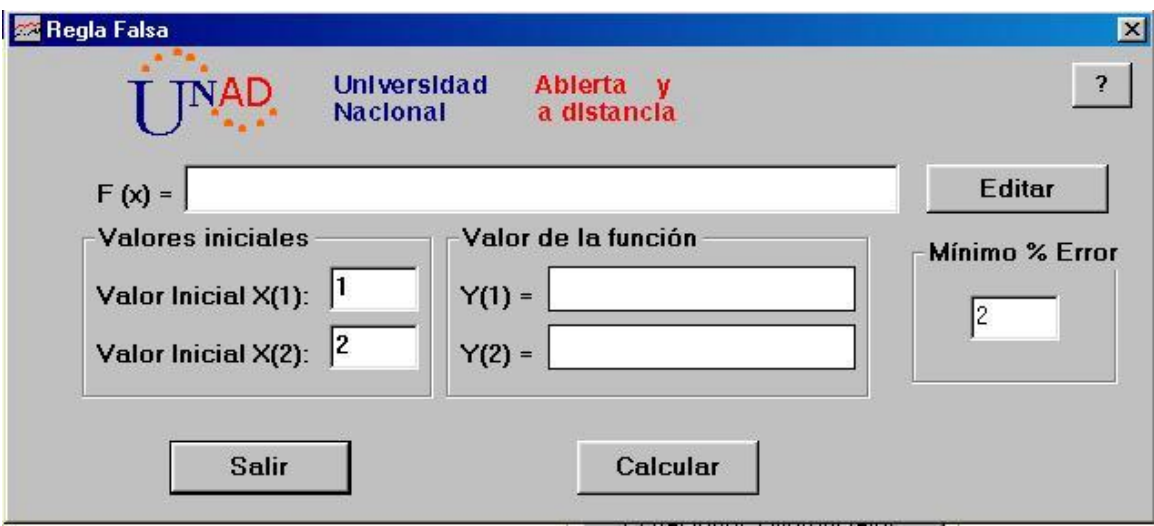

(b)

Figura 5. Ventanas para acceder a los métodos de solución de ecuaciones no lineales: (a) Newton - Raphson, (b) Regla Falsa.

La solución se expresa en un formulario de respuesta con el objeto de reconocer las diferencias entre los métodos de intervalos (Bisección, Falsa posición,...), de los métodos abiertos (Newton-Raphson, Secante,...), así:

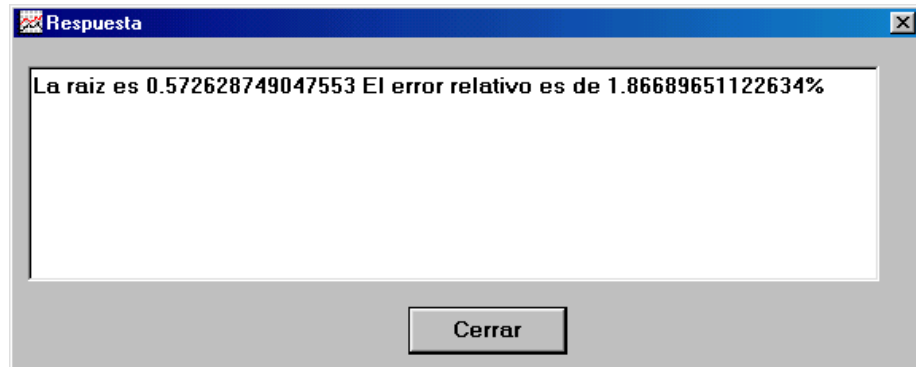

Figura 6. Ventana que presenta los resultados en los cálculos de solución de ecuaciones no lineales. 


\section{Integración}

Tal como se representa, una interpretación física de la integración numérica es la determinación del área bajo la curva. La integración tiene muchas aplicaciones en la práctica de la ingeniería, empezando por la determinación de los centroides de objetos de formas extravagantes, hasta el cálculo de cantidades totales basados en conjuntos de medidas discretas. Adicionalmente, las formulas de integración numérica juegan un papel importante en la solución de las ecuaciones diferenciales.

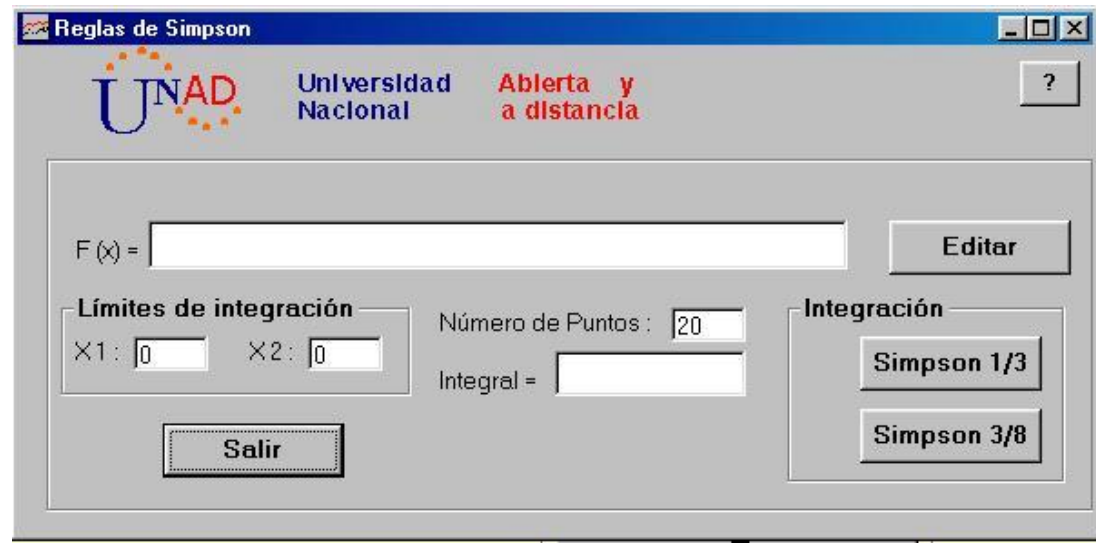

(c)

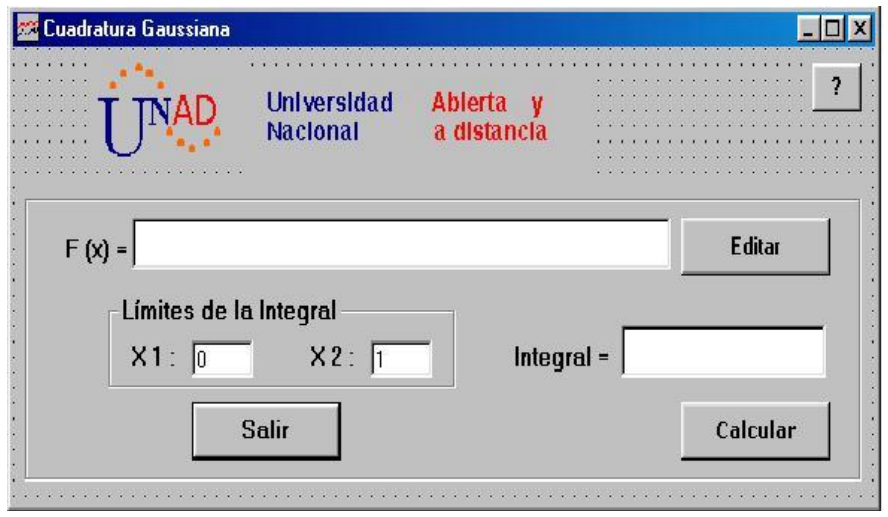

(d)

Figura 7. Ventana que presenta los resultados para los métodos de integración: (c) Reglas de Simpson, (d) Cuadratura Gaussiana.

Con frecuencia surge la necesidad de evaluar la integral definida de una función que no tiene una antiderivada explicita o cuya antiderivada tiene valores que no son fácilmente obtenibles. El método involucrado para aproximar $\int \mathrm{F}(\mathrm{x}) \mathrm{dx}$ se conoce como cuadratura numérica y usa una suma del tipo $\sum \mathrm{a}_{\mathrm{i}} \mathrm{F}\left(\mathrm{x}_{\mathrm{i}}\right)$ para aproximar $\int \mathrm{F}(\mathrm{x}) \mathrm{dx}$.

\section{Ajuste de curvas}

A menudo se presentará la oportunidad de ajustar curvas a un conjunto de datos representados por puntos. 


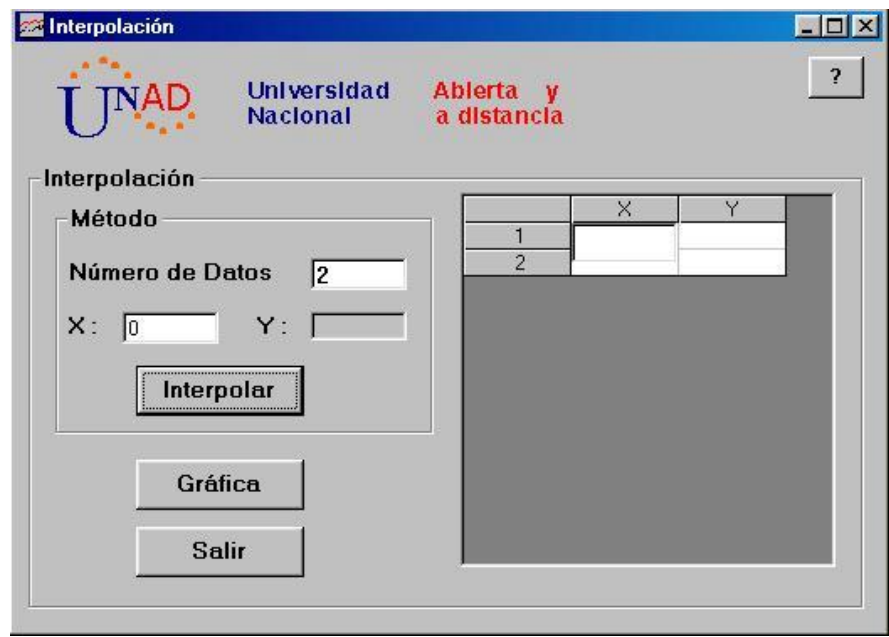

(e)

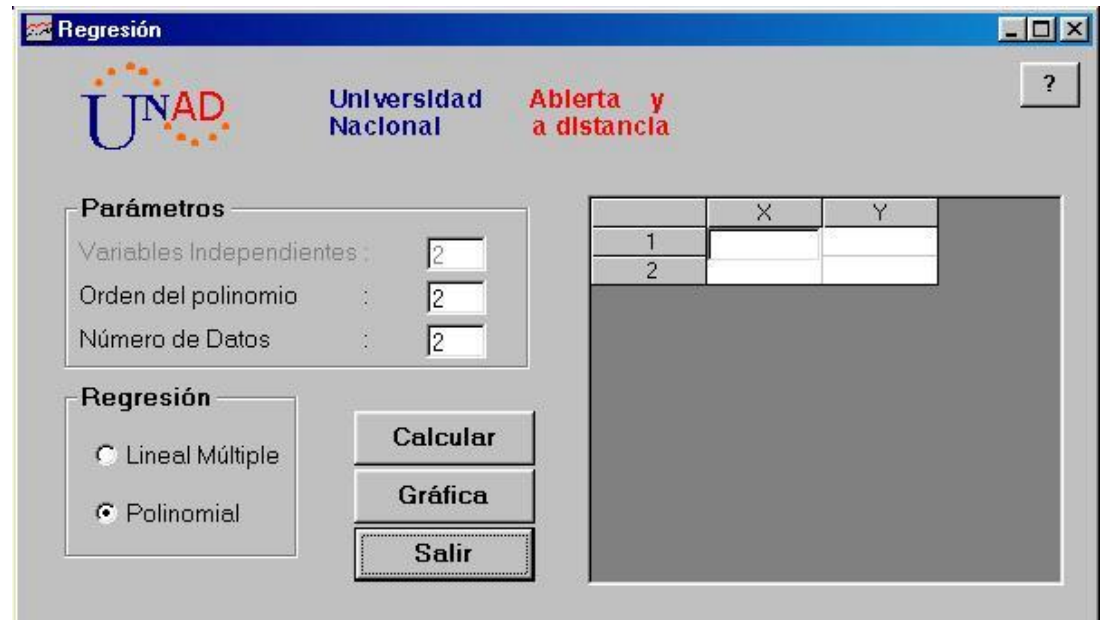

(f)

Figura 8. En la pantalla de la computadora se muestran la gráfica de datos transformados que se usan para determinar los coeficientes de la ecuación de potencias: (e) Interpolación, (f) Regresión.

Las técnicas que se han desarrollado para este fin pueden dividirse en dos categorías generales: regresión e interpolación. La primera se emplea cuando hay un grado significativo de error asociado a los datos; con frecuencia los datos experimentales son de esta clase. Para esta estrategia es encontrar una curva que represente la tendencia general de los datos sin necesidad de tocar los puntos individuales. En contraste, la interpolación se maneja cuando el objetivo es determinar valores intermedios entre datos que estén, relativamente libres de error. Tal es el caso de la información tabulada. Para estas situaciones, la estrategia es ajustar una curva directamente mediante los puntos y usar esta curva para predecir valores intermedios. 


\section{Ecuaciones Simultáneas}

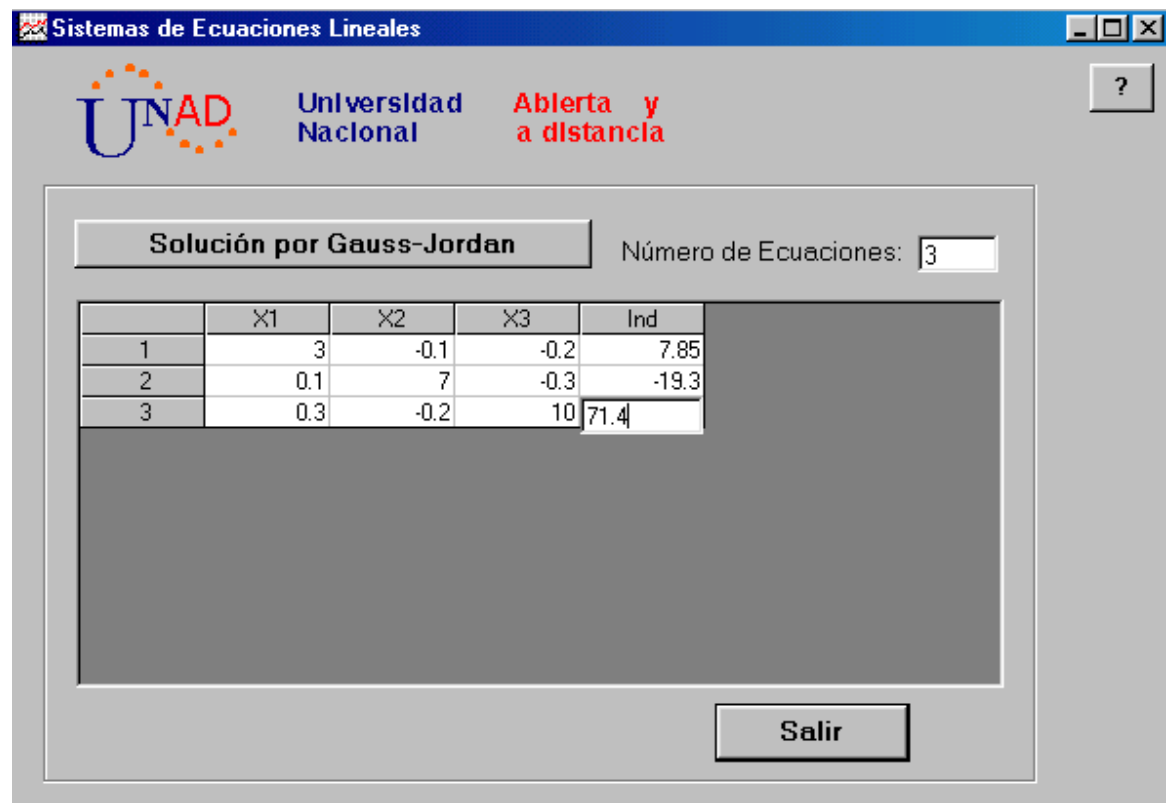

$(\mathrm{g})$

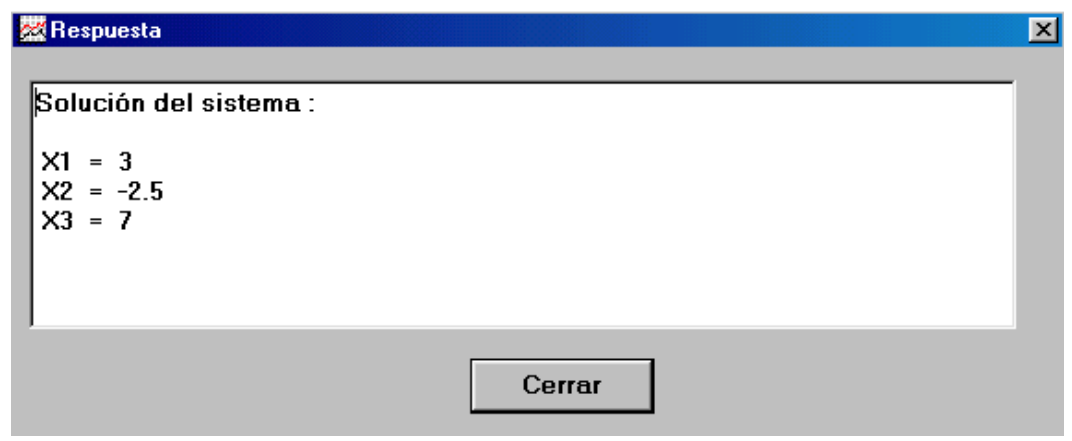

(h)

Figura 9. Ejemplo de aplicación del software para un caso de ecuaciones simultáneas (g) Formulario de entrada de datos (h) Ventana de respuesta.

Los sistemas lineales de ecuaciones se presentan en muchos problemas de ingeniería y de ciencia, así como en las aplicaciones de las matemáticas a las ciencias sociales y al estudio cuantitativo de problemas de comercio y de economía. En particular, se originan a partir de modelos matemáticos de sistemas grandes de elementos interrelacionados, como: estructuras, circuitos eléctricos y redes de flujo; aunque también pueden encontrarse en otras áreas de los métodos numéricos como el ajuste de curva y ecuaciones diferenciales. 


\section{Ecuaciones no Lineales}

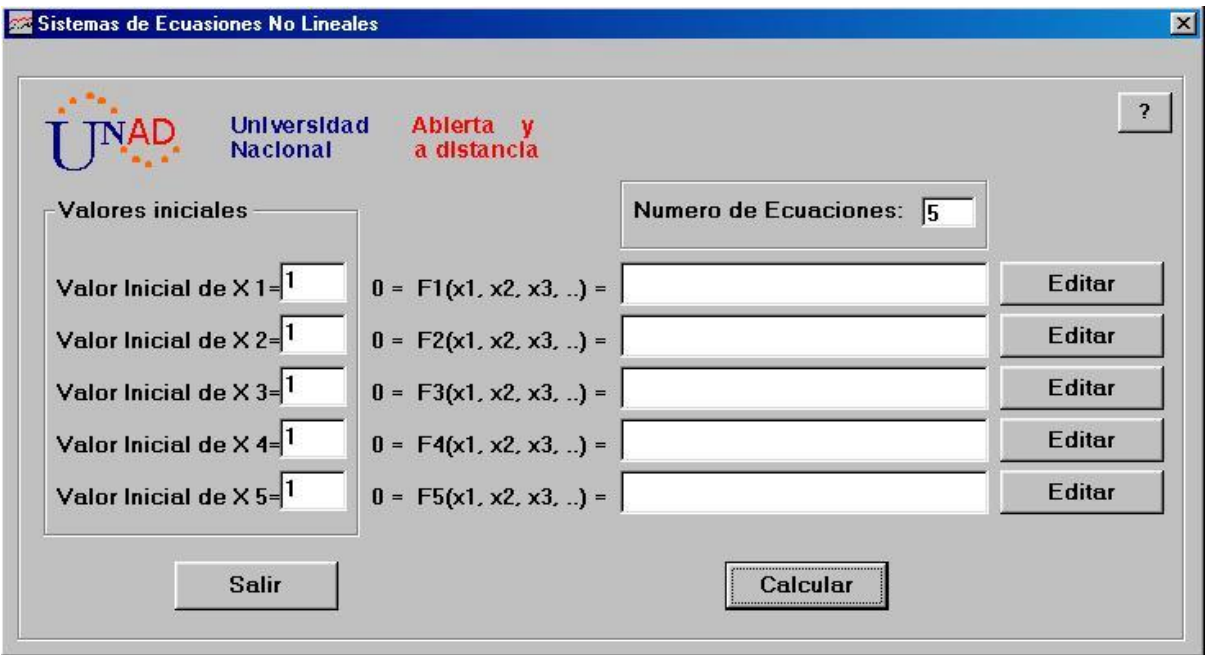

Figura 10. Formulario de acceso para resolver numéricamente un sistema de ecuaciones no lineales.

Los métodos de iteración de punto fijo dominan el estudio de las soluciones numéricas de ecuaciones de la forma $\mathrm{F}(\mathrm{x})=0$. El método que usaremos para resolver sistemas de ecuaciones no lineales será básicamente una generalización del método de Newton.

\section{Ecuaciones Diferenciales}

Aunque en cualquier libro de texto sobre el tema de ecuaciones diferenciales ordinarias se encuentran métodos para hallar explícitamente las soluciones de problemas de primer orden de valor inicial, en la práctica pocos de los problemas que se originan del estudio de fenómenos físicos se pueden resolver exactamente. Estas tienen un enorme significado en la práctica de la ingeniería. Esto se debe a que muchas leyes físicas están expresadas en términos de la razón de cambio de una cantidad más que en términos de su magnitud. Entre los ejemplos se observan desde los modelos de predicción demográfica (razón de cambio de la población) hasta la aceleración de un cuerpo en descenso (razón de cambio de la velocidad). Dos tipos de problemas son direccionados: problemas con valor inicial y valores en la frontera. 


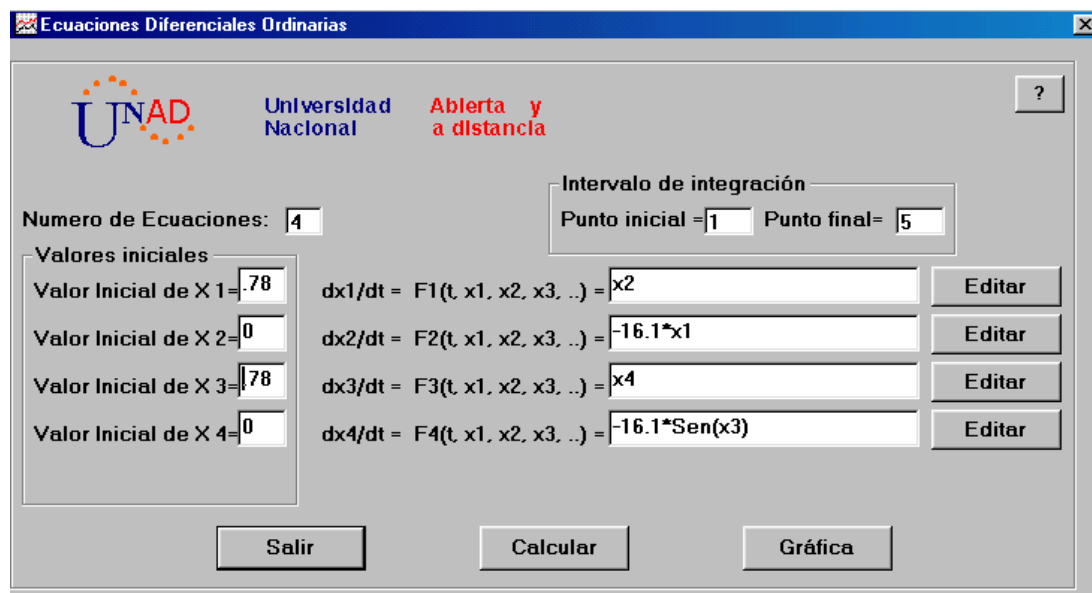

(i)

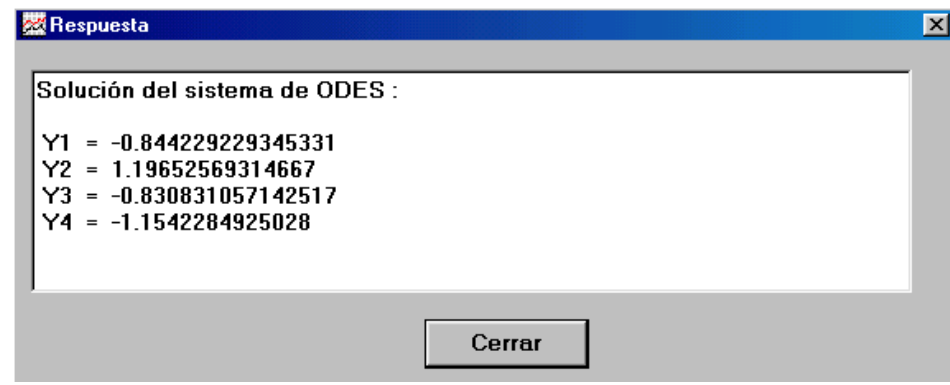

(j)

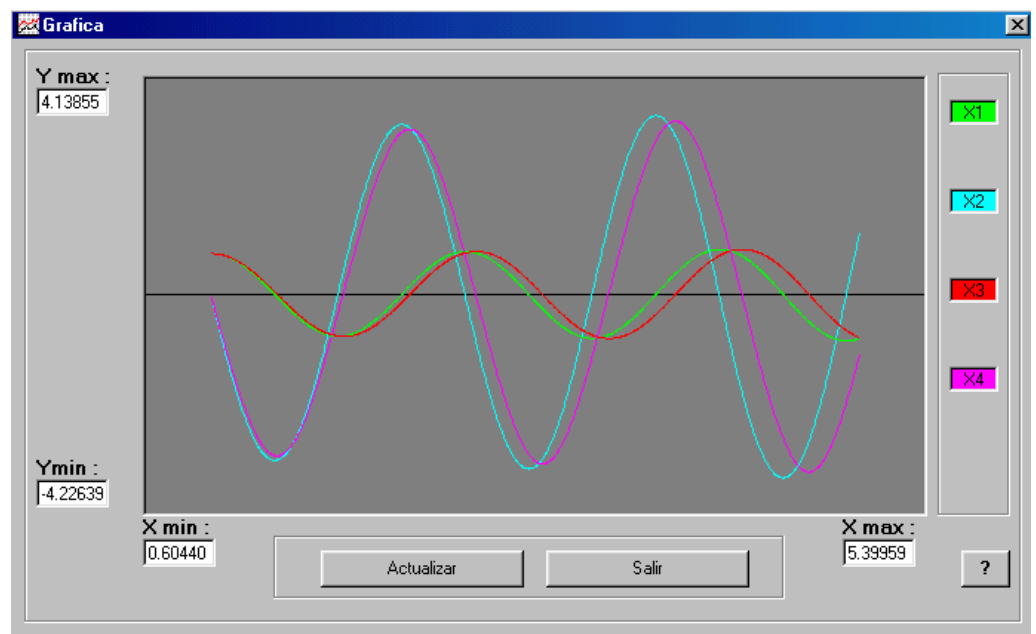

(k)

Figura 11. Pantallas para la resolución de problemas de valor inicial para ecuaciones diferenciales ordinarias. (i) presentación y captura de datos (j) Ventana de respuesta (k) Solución grafica. 


\section{Depuración de Errores}

Durante la ejecución de algunos formularios, se le presentarán algunos mensajes de ayuda, cuando se han realizado errores, como se muestra a continuación:

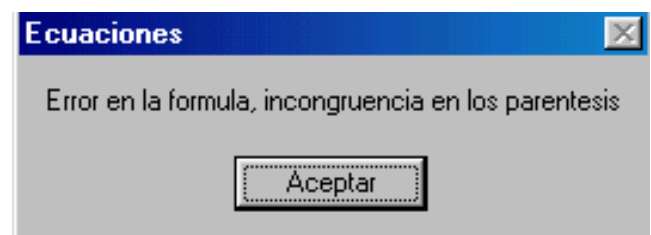

Error en la formula digitada en el editor de ecuaciones.

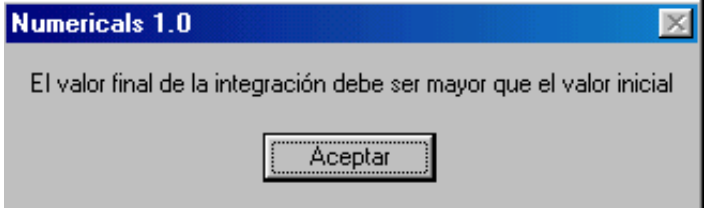

Error por desconocimiento de la teoría básica para el manejo de la técnica numérica.

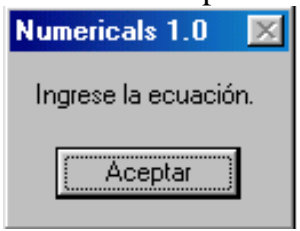

Uso erróneo de la función o sentencia, se intento usar un arreglo sin declarar.

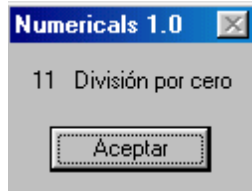

Error matemático tal como la división por cero o el argumento excede el rango de cálculo permisible.

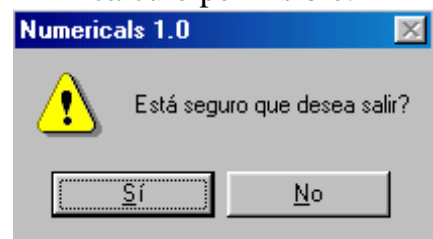

Con la tecla «Esc» del teclado de su computadora puede llamar el mensaje de «Salir» permanentemente desde cualquier formulario.

Figura 12. Ventanas de ayuda para indicar los errores cometidos en los cálculos.

\section{Recomendaciones}

El Software Tutorial Numericals 1.0 fue desarrollado con el propósito de implementar el Laboratorio para el curso de Análisis Numérico y como tal, no sólo se presenta como material exclusivo del curso sino que además puede surgir interés en aumentar este trabajo incrementando nuevos métodos que le darían al software un mayor alcance, para este fin, el Tutorial está disponible a las actualizaciones que se requieran. 


\section{Bibliografía}

[Dd Bakahvalov, N., Métodos Numéricos, Editorial Paraninfo, Madrid, 1990.

[Da Balfour, A. y Beveridge, W. T., Análisis Numérico Básico con Algol. Compañía Editorial Continental, S.A., Primera edición, México, 1987.

[D] Burden, Richard y otros, Análisis Numérico, Grupo Editorial Iberoaméricana, México, 1985.

[D] Chapra, Steven C. y Canale, Raymond P., Métodos Numéricos para Ingenieros, con aplicaciones en computadoras personales. McGraw-Hill, S. A. de C. V., Primera edición, México, 1988.

[D] Cutlip, Michael B. y Schacham, Mordechai, Problem Engineering in Chemical Engineering with Numerical Methods. Prentice Hall International, 1st Edition, New York, 1999.

[D] Enciclopedia Microsoft Encarta®2001. @1993-2000 Microsoft Corporation. Reservados todos los derechos.

[Dd Hildebrand, F. B., Introduction to Numerical Analysis, Second Edition, McGrawHill, New York, 1974.

[D] Merayo G., Félix y Nevot L., Antonio, Análisis Numérico, Editorial Paraninfo, Primera edición, Madrid, 1992.

[C] Nakamura, Shoichiro, Análisis numérico y visualización gráfica con MATLAB, Prentice-Hall Hispanomericana, S.A. Primera edición, México, 1997.

[D] $\square$ Purcell, Edwing J. y Varberg, Dale, Cálculo con Geometría Analítica, PrenticeHall Hispanoamericana S. A., Cuarta edición, México, 1987. 